\title{
A GENERAL FIBRE-REINFORCED COMPOSITE SHELL ELEMENT BASED ON A REFINED SHEAR DEFORMATION THEORY
}

\author{
MallikarJuna $\dagger$ and T. Kant $\ddagger$ \\ Department of Civil Engineering, Indian Institute of Technology, IIT-Powai, Bombay 400 076, India
}

(Received 31 October 1990)

\begin{abstract}
A refined shell theory has been developed for the analysis of isotropic, orthotropic and anisotropic fibre-reinforced laminated composite and sandwich shells. This theory is based on a higher-order displacement model and the three-dimensional Hooke's laws for shell material, giving rise to a more realistic representation of the cross-sectional deformation. The superparametric shell element with four-noded linear eight/nine-noded quadratic and twelve/sixteen-noded cubic, serendipity/ Lagrangian shape functions can be employed. In addition to the present higher-order shear deformation shell theory (HOST), a first-order shear deformation shell theory (FOST), following Reissner-Mindlin plate's formulation, is developed and the results are compared with the closed-form solutions (CFS). The parametric effects of the finite element mesh, radius-to-arc length ratio, arc length-to-thickness ratio, lamination scheme, Gaussian integration rule, and material anisotropy on the response of the laminated composite shells are investigated. Results are tabulated to provide an easy means for future comparisons by other investigators.
\end{abstract}

\section{INTRODUCTION}

The evolution of the technology of structural materials has been governed by the search for higher strength-to-weight ratios, lower costs, ease of fabrication and better durability. The development of the aeroplane with its peculiar requirement of structures which needed to be strong but light and flexible but tough brought into being a new breed of hybrid structural materials called fibre-reinforced composites. The increased use of composites for high performance design applications necessitates the need for a more realistic prediction of the response characteristics of composite material structures. Due to low shear modulus relative to in-plane Young's modulus, transverse shear deformation effects are more pronounced in composite laminates. Thus, reliable prediction of the response characteristics of high modulus composites requires the use of shear deformable theories.

Several theories to analyse shells have been put forward in which the effects of shear deformation have been taken into account [1-7]. An exposition of various shell theories is available in [8]. Although the above mentioned shear deformation theories yield acceptable solutions, they are found to be inadequate to predict the accurate response characteristics of anisotropic composite-sandwich shells. The analysis of such problems requires the use of refined theories [9-15]. More recently, the present authors [16-20] have established the higher-order

† Presently at Civil Engineering Department, Université Laval, Québec, Canada G1K 7P4.

$\ddagger$ To whom all correspondence should be addressed. theories for composite-sandwich plates in conjunction with simple $C^{0}$ isoparametric finite element formulation. In the present investigation a refined higher-order displacement model is used with the superparametric shell element for analysis of general anisotropic shell structures. A detailed description of the element following Ahmad et al. [21], Belytschko et al. [22], Milford and Schnobrich [23], and Kant and Datye [24] is presented.

\section{FINITE ELEMENT DISCRETIZATION}

The following sections present the development of a refined theory with the general shell element.

\section{Geometric definition of the element}

Elements with a higher degree of interpolation on the coordinates than on the displacements are called, in general, superparametric elements. In the present formulation, three types of coordinates are employed: nodal coordinates $\left(V_{1}, V_{2}, V_{3}\right)$, local coordinates $\left(x^{\prime}, y^{\prime}, z^{\prime}\right)$ and global coordinates $(x, y, z)$. A typical quadrilateral shell element is considered. The external faces of the element are curved, while the sections across the thickness are generated by straight lines. The boundaries of the element are defined by nondimensional coordinates $\xi, \eta= \pm 1$ and $z^{\prime}= \pm t / 2$. The coordinates of any point $\left(\xi, \eta, z^{\prime}\right)$ can be expressed as

$$
\left\{\begin{array}{l}
x \\
y \\
z
\end{array}\right\}=\sum_{i=1}^{N N} N_{i}\left\{\begin{array}{l}
x_{i} \\
y_{i} \\
z_{i}
\end{array}\right\}_{\text {mid }}+\sum_{i=1}^{N N} N_{i} z^{\prime} \mathbf{V}_{3 i}
$$


in which $N N$ denotes the number of nodes in an element, $N_{i}$ is the shape function of $i$ th node of the four-noded bi-linear or eight-noded serendipity/ninenoded Langrangian quadrilateral element, $\left(x_{i}, y_{i}, z_{i}\right)$ are the midsurface global coordinates of the $i$ th nodal point which is computed by taking the average of top and bottom coordinates. Vector $\mathbf{V}_{3 i}$ is defined by input data, and is presumed to span the thickness and be normal to the midsurface. Vector $\mathbf{V}_{1 i}$ is of arbitary length and might also be defined by input data so that is coincides with a principal direction of an orthotropic material. Or, $\mathbf{V}_{1 i}$ could be defined by the program itself as the cross product $\mathbf{V}_{14}=\mathbf{V}_{3 i} \times \overline{\mathbf{i}}$ or $\mathbf{V}_{1 i}=\mathbf{V}_{3 i} \times \hat{\mathbf{j}}$, where $\hat{\mathbf{i}}$ and $\mathbf{j}$ are unit vectors in $x$ - and $y$-directions. The vector $\mathbf{V}_{2 i}$ is perpendicular to the plane defined by $\mathbf{V}_{3 i}$ and $\mathbf{V}_{1 i}$.

The local coordinate set $\left(x^{\prime}, y^{\prime}, z^{\prime}\right)$ is a Cartesian coordinate system defined at the sampling points wherein stresses and strains are to be calculated. At any point $(\xi, \eta)$ on the midsurface, an orthogonal set of local coordinate axes $\left(x^{\prime}, y^{\prime}, z^{\prime}\right)$ are constructed. $z^{\prime}$ is the normal unit vector and $\hat{\mathbf{x}}^{\prime}$ and $\hat{\mathbf{y}}^{\prime}$ are tangent to the midsurface. If $\hat{\mathbf{V}}_{\xi}$ and $\hat{\mathbf{V}}_{\eta}$ be unit vectors along $\xi$ and $\eta$-directions, respectively, then

$$
\hat{\mathbf{z}}=\frac{\mathbf{V}_{\xi} \times \mathbf{V}_{\eta}}{\left|\mathbf{V}_{\xi} \times \mathbf{V}_{\eta}\right|},
$$

where

$$
\begin{aligned}
& \mathbf{V}_{\xi}=\frac{\partial x}{\partial \xi} \hat{\mathbf{i}}+\frac{\partial y}{\partial \xi} \hat{\mathbf{j}}+\frac{\partial z}{\partial \xi} \mathbf{k} \\
& \mathbf{V}_{\eta}=\frac{\partial x}{\partial \eta} \hat{\mathbf{i}}+\frac{\partial y}{\partial \eta} \hat{\mathbf{j}}+\frac{\partial z}{\partial \eta} \mathbf{k} .
\end{aligned}
$$

In order to define the material directions for anisotropic structures in relation to the local system of axes in a consistent manner, a definition similar to that of the $\mathbf{V}_{1 i}$-direction is adopted here.

The direction cosine matrices $\boldsymbol{\mu}_{i}$ and $\boldsymbol{\mu}^{\prime}$, which relate the transformations between the nodal and global, and local and global coordinate system, respectively, are given by,

$$
\boldsymbol{\mu}_{i}=\left[\begin{array}{ccc}
l_{1 i} & l_{2 i} & l_{3 i} \\
m_{1 i} & m_{3 i} & m_{3 i} \\
n_{1 i} & n_{2 i} & n_{3 i}
\end{array}\right] \text { and } \boldsymbol{\theta}^{\prime}=\left[\begin{array}{ccc}
l_{1} & l_{2} & l_{3} \\
\bar{m}_{1} & \bar{m}_{2} & \bar{m}_{3} \\
\bar{n}_{1} & \bar{n}_{2} & \bar{n}_{3}
\end{array}\right] \text {, }
$$

where $l, m, n$ represent direction cosines. These individual transformation matrices are orthogonal.

In the present model, the Jacobian relates the area integral in the $x^{\prime}, y^{\prime}$-coordinates to that in the $\xi, \eta$ coordinates, which is given by

$$
\mathbf{J}=\left[\begin{array}{ll}
\mathbf{V}_{\xi} \cdot \hat{\mathbf{x}}^{\prime} & \mathbf{V}_{\xi} \cdot \hat{\mathbf{y}}^{\prime} \\
\mathbf{V}_{\eta} \cdot \hat{\mathbf{x}}^{\prime} & \mathbf{V}_{\eta} \cdot \hat{\mathbf{y}}^{\prime}
\end{array}\right]=\left[\begin{array}{ll}
J_{11} & J_{12} \\
J_{21} & J_{22}
\end{array}\right]
$$

with $\mathbf{J}^{*}=\mathbf{J}^{-1}$, the following relations are used subsequently

$$
\begin{aligned}
\frac{\partial}{\partial x^{\prime}} & =J_{11}^{*} \frac{\partial}{\partial \xi}+J_{12}^{*} \frac{\partial}{\partial \eta} \\
\frac{\partial}{\partial y^{\prime}} & =J_{21}^{*} \frac{\partial}{\partial \xi}+J_{22}^{*} \frac{\partial}{\partial \eta}
\end{aligned}
$$

\section{HIGHER-ORDER DISPLACEMENT MODEL}

The element displacements are completely defined by the midsurface nodal degrees of freedom $u_{i}, v_{i}$ and $w_{i}$ in the $x, y$ and $z$ global coordinate directions, and by rotations $\beta_{i}, \alpha_{i}$ and $\gamma_{i}$ about $V_{1 i}, V_{2 i}$ and $V_{3 i}$ axes, respectively; $\beta_{i}^{*}, \alpha_{i}^{*}$ and $\gamma_{i}^{*}$ are the corresponding higher-order degrees of freedom. Vectors $\mathbf{V}_{1 i}, \mathbf{V}_{2 i}, \mathbf{V}_{3 i}$ are mutually perpendicular. The displacements at any point $\left(\xi, \eta, z^{\prime}\right)$ can be expressed in terms of the nodal displacements as

$$
\begin{aligned}
\left\{\begin{array}{l}
u \\
v \\
w
\end{array}\right\}=\sum_{i=1}^{N N} N_{i}\left\{\begin{array}{l}
u_{i} \\
v_{i} \\
w_{i}
\end{array}\right\}+\sum_{i=1}^{N N} N_{i} z^{\prime} \mu_{k}\left\{\begin{array}{c}
\alpha_{i} \\
-\beta_{i} \\
-\gamma_{i}
\end{array}\right\} \\
+\sum_{i=1}^{N N} N_{i} z^{\prime 2} \mu_{i}\left\{\begin{array}{c}
\alpha_{i}^{*} \\
-\beta_{i}^{*} \\
-\gamma_{i}^{*}
\end{array}\right\} .
\end{aligned}
$$

The element coordinate definition given by the relation (1) has more degrees of freedom than the definition of the displacements. The element is therefore of the superparametric kind. The displacements $\left(u_{i}, v_{i}, w_{i}\right),\left(\alpha_{i}, \beta_{i}, \gamma_{i}\right),\left(\alpha_{i}^{*}, \beta_{i}^{*}, \gamma_{i}^{*}\right)$ and $\left(u^{\prime}, v^{\prime}, w^{\prime}\right)$, $\left(\theta_{x^{\prime}}, \theta_{y^{\prime}}, \theta_{z^{\prime}}\right),\left(\theta_{x^{\prime}}^{*}, \theta_{y}^{*}, \theta_{z^{\prime}}^{*}\right)$ in the nodal and local coordinate systems, respectively, can be expressed in terms of global coordinate system by using expressions (3a) and (3b), respectively.

\section{ELEMENT STIFFNESS MATRIX}

Since the strain-displacement matrix $B$ and the rigidity matrix $\mathbf{D}$ are being obtained in local coordinates, the same matrices are used for the computation of element stiffness matrix. Here the transformation is derived based on the concept that during any virtual displacement, the resulting increment in strain energy density must be the same regardless of the coordinate system in which it is computed. The relationship between the strains at any point within a laminate and the corresponding deformations are the functions of the assumed displacement field. In $C^{0}$ finite element, the continuum displacement vector within the element is discretized such that

$$
\boldsymbol{\delta}=\sum_{i=1}^{N N} N_{i} \boldsymbol{\delta}_{i}
$$

in which $\boldsymbol{\delta}_{i}$ is the generalized displacement vector 
corresponding to the $i$ th node of an element. The generalized strain vector $\bar{\epsilon}^{\prime}$ at any point within an element in the local coordinate system can be expressed by the following relationships

$$
\overrightarrow{\boldsymbol{\epsilon}}^{\prime}=\sum_{i=1}^{N N} \mathbf{B}_{i} \boldsymbol{\delta}_{i}
$$

where

$$
\begin{gathered}
\bar{\epsilon}^{\prime}=\left[\epsilon_{x_{0}^{\prime}}, \epsilon_{y_{0}^{\prime}}, \epsilon_{z_{0}^{\prime}}, \gamma_{x^{\prime} y_{0}^{\prime}}, \chi_{x^{\prime}}, \chi_{y^{\prime}}, \chi_{z^{\prime}} \chi_{x^{\prime} y^{\prime}}, \epsilon_{x_{0}^{\prime}}^{*},\right. \\
\left.\epsilon_{y_{0}^{\prime}}^{*}, \gamma_{x^{\prime} y_{0}^{\prime}}^{*} \gamma_{x^{\prime} z_{0}^{\prime}}, \gamma_{y^{\prime} z_{0}^{\prime}}, \phi_{x^{\prime}}, \phi_{y^{\prime}}, \gamma_{x^{\prime} z_{0}^{\prime}}^{*}, \gamma_{y^{\prime} z_{0}^{\prime}}^{*}\right] \\
\delta_{i}=\left[u_{i}, v_{i}, w_{i}, \alpha_{i}, \beta_{i}, \gamma_{i}, \alpha_{i}, \beta_{i}^{*}, \gamma_{i}^{*}\right]^{T}
\end{gathered}
$$

B is the strain-displacement matrix defined in terms of the displacement derivatives with respect to the local Cartesian coordinates and non-zero terms of this matrix are given below:

(i) Membrane, coupling between inplane and bending and flexure terms

$$
\begin{aligned}
& B_{11}=\left(\partial N_{i} / \partial x^{\prime}\right) I_{1}, \quad B_{12}=\left(\partial N_{i} / \partial x^{\prime}\right) \bar{m}_{1} \\
& B_{13}=\left(\partial N_{i} / \partial x^{\prime}\right) \bar{n}_{1}, \quad B_{21}=\left(\partial N_{i} / \partial y^{\prime}\right) I_{2}, \\
& B_{22}=\left(\partial N_{1} / \partial y^{\prime}\right) \bar{m}_{2}, \quad B_{23}=\left(\partial N_{i} / \partial y^{\prime}\right) \bar{n}_{2}, \\
& B_{34}=-N_{i} E_{32 i}, \quad B_{35}=-N_{i} E_{31 i}, \quad B_{36}=-N_{i} E_{33 i}, \\
& B_{41}=\left(\partial N_{1} / \partial x^{\prime}\right) I_{2}+\left(\partial N_{i} / \partial y^{\prime}\right) I_{1}, \\
& B_{42}=\left(\partial N_{i} / \partial x^{\prime}\right) \bar{m}_{2}+\left(\partial N_{\imath} / \partial y^{\prime}\right) \bar{m}_{1} \\
& B_{43}=\left(\partial N_{i} / \partial x^{\prime}\right) \bar{n}_{2}+\left(\partial N_{i} / \partial y^{\prime}\right) \tilde{n}_{1} \\
& B_{54}=\left(\partial N_{i} / \partial x^{\prime}\right) E_{22 !}, \quad B_{55}=\left(\partial N_{i} / \partial x^{\prime}\right) E_{21 i}, \\
& B_{56}=\left(\partial N_{1} / \partial x^{\prime}\right) E_{23 i}, \quad B_{64}=-\left(\partial N_{i} / \partial y^{\prime}\right) E_{12 i}, \\
& B_{65}=-\left(\partial N_{i} / \partial y^{\prime}\right) E_{11 i}, \quad B_{66}=-\left(\partial N_{i} / \partial y^{\prime}\right) E_{13 i}, \\
& B_{77}=-2 N_{i} E_{32 i}, \quad B_{78}=-2 N_{t} E_{31 l} \\
& B_{79}=-2 N_{i} E_{33 i} \\
& B_{84}=\left(\partial N_{i} / \partial y^{\prime}\right) E_{22 i}-\left(\partial N_{i} / \partial x^{\prime}\right) E_{12 i}, \\
& B_{85}=\left(\partial N_{i} / \partial y^{\prime}\right) E_{21 i}-\left(\partial N_{i} / \partial x^{\prime}\right) E_{11 i} \\
& B_{86}=\left(\partial N_{i} / \partial y^{\prime}\right) E_{23 i}-\left(\partial N_{i} / \partial x^{\prime}\right) E_{13 t} \\
& B_{97}=\left(\partial N_{i} / \partial x^{\prime}\right) E_{22 i}, \quad B_{98}=\left(\partial N_{i} / \partial x^{\prime}\right) E_{21 i} \\
& B_{9,9}=\left(\partial N_{i} / \partial x^{\prime}\right) E_{23 i}, \quad B_{10,7}=-\left(\partial N_{i} / \partial y^{\prime}\right) E_{12 i}, \\
& B_{10,8}=-\left(\partial N_{i} / \partial y^{\prime}\right) E_{11 i}, \quad B_{10,9}=-\left(\partial N_{i} / \partial y^{\prime}\right) E_{13 i} \\
& B_{11,7}=\left(\partial N_{i} / \partial y^{\prime}\right) E_{22 i}-\left(\partial N_{i} / \partial x^{\prime}\right) E_{12 i},
\end{aligned}
$$

$B_{11,8}=\left(\partial N_{i} / \partial y^{\prime}\right) E_{21 i}-\left(\partial N_{i} / \partial x^{\prime}\right) E_{11 i}$

$B_{11,9}=\left(\partial N_{i} / \partial y^{\prime}\right) E_{23 i}-\left(\partial N_{i} / \partial x^{\prime}\right) E_{13 i}$

(ii) Shear terms

$B_{12,1}=\left(\partial N_{i} / \partial x^{\prime}\right) I_{3}, \quad B_{12,2}=\left(\partial N_{i} / \partial x^{\prime}\right) \bar{m}_{3}$,

$B_{12,3}=\left(\partial N_{i} / \partial x^{\prime}\right) \tilde{n}_{3}, \quad B_{12,4}=N_{i} E_{22 i}$,

$B_{12,5}=N_{i} E_{21 i}, \quad B_{12,6}=N_{i} E_{23 i}$,

$B_{13,1}=\left(\partial N_{i} / \partial y^{\prime}\right) I_{3}, \quad B_{13,2}=\left(\partial N_{i} / \partial y^{\prime}\right) \bar{m}_{3}$,

$B_{13,3}=\left(\partial N_{i} / \partial y^{\prime}\right) \bar{n}_{3}, \quad B_{13,4}=-N_{i} E_{12 i}$,

$B_{13,5}=-N_{i} E_{11 i}, \quad B_{13,6}=-N_{i} E_{13 i}$

$B_{14,4}=-\left(\partial N_{i} / \partial x^{\prime}\right) E_{32 i}, \quad B_{14,5}=-\left(\partial N_{i} / \partial x^{\prime}\right) E_{31 i}$

$B_{14,6}=-\left(\partial N_{i} / \partial x^{\prime}\right) E_{33 i} \quad B_{14,7}=2 N_{i} E_{22 i}$,

$B_{14,8}=2 N_{i} E_{21 i}, \quad B_{14,9}=2 N_{i} E_{23 i}$,

$B_{15,4}=-\left(\partial N_{i} / \partial y^{\prime}\right) E_{32 i}, \quad B_{15,5}=-\left(\partial N_{i} / \partial y^{\prime}\right) E_{31 i}$

$B_{15,6}=-\left(\partial N_{1} / \partial y^{\prime}\right) E_{33 i}, \quad B_{15,7}=-2 N_{i} E_{12 i}$,

$B_{15,8}=-2 N_{i} E_{11 i}, \quad B_{15,9}=-2 N_{i} E_{13 i}$

$B_{16,7}=-\left(\partial N_{i} / \partial x^{\prime}\right) E_{32 i}, \quad B_{16,8}=-\left(\partial N_{i} / \partial x^{\prime}\right) E_{31 i}$,

$B_{16,9}=-\left(\partial N_{i} / \partial x^{\prime}\right) E_{33 i}, \quad B_{17,7}=-\left(\partial N_{i} / \partial y^{\prime}\right) E_{32 i}$

$B_{17,8}=-\left(\partial N_{i} / \partial y^{\prime}\right) E_{31 i}, \quad B_{17,9}=-\left(\partial N_{i} / \partial y^{\prime}\right) E_{331}$,

where

$$
\begin{gathered}
\frac{\partial N_{i}}{\partial x^{\prime}}=J_{11}^{*} \frac{\partial N_{i}}{\partial \xi}+J_{12}^{*} \frac{\partial N_{i}}{\partial \eta} \\
\frac{\partial N_{i}}{\partial y^{\prime}}=J_{21}^{*} \frac{\partial N_{i}}{\partial \xi}+J_{22}^{*} \frac{\partial N_{i}}{\partial \eta} \\
E_{11 i}=I_{1} l_{l i}+\bar{m}_{1} m_{1 i}+\bar{n}_{1} n_{1 i}, \\
E_{12 i}=I_{1} l_{2 i}+\bar{m}_{1} m_{2 i}+\bar{n}_{1} n_{2 i} \\
E_{13 i}=I_{1} l_{3 i}+\bar{m}_{1} m_{3 i}+\bar{n}_{1} n_{3 i}, \\
E_{21 i}=I_{2} l_{l i}+\bar{m}_{2} m_{1 i}+\bar{n}_{2} n_{1 i} \\
E_{22 i}=I_{2} l_{2 i}+\bar{m}_{2} m_{2 i}+\bar{n}_{2} n_{2 i}, \\
E_{23 i}=I_{2} l_{3 i}+\bar{m}_{2} m_{3 i}+\bar{n}_{2} n_{3 i}, \\
E_{31 i}=I_{3} l_{l i}+\bar{m}_{3} m_{1 i}+\bar{n}_{3} n_{l i}, \\
E_{32 i}=I_{3} l_{2 i}+\bar{m}_{3} m_{2 i}+\bar{n}_{3} n_{2 i}, \\
E_{33 i}=I_{3} l_{3 i}+\bar{m}_{3} m_{3 i}+\bar{n}_{3} n_{3 i} .
\end{gathered}
$$


If the reference system of orthogonal axes $\left(x^{\prime}, y^{\prime}, z^{\prime}\right)$ is parallel to the principal material axes $(1,2,3)$, then the stress-strain relationships can be written as

$$
\sigma_{1,2,3}=\mathbf{C} \epsilon_{1,2,3} \quad \text { or } \quad \sigma_{x^{\prime}, y^{\prime}, z^{\prime}}=\mathbf{C} \boldsymbol{\epsilon}_{x^{\prime}, y^{\prime}, z^{\prime}} .
$$

If the principal axes of anisotropy 1,2 do not coincide with the reference axes $x^{\prime} y^{\prime}$, but are rotated by a certain angle $\theta$, the new elasticity matrix $\mathbf{Q}$ is determined from the following transformation

$$
\mathbf{Q}=\mathbf{T}^{-1} \mathbf{C} \mathbf{T}^{-1 T},
$$

where the superscript $T$ denotes the matrix transpose, $\mathbf{T}$ is the transformation matrix and $\mathbf{C}$ and $\mathbf{Q}$ are used here to denote the reduced stiffness matrix and transformed reduced stiffness matrix, and all the above matrices are given in [16]. The stress-strain relations in $x^{\prime} y^{\prime}$-coordinates can be written as

$$
\boldsymbol{\sigma}_{x^{\prime} y^{\prime} z^{\prime}}=\mathbf{Q} \epsilon_{x y^{\prime} z^{\prime} z^{\prime}}
$$

The constitutive relations involving inplane forces, bending moments and shear forces are defined as follows: inplane and bending, and flexure rigidities, and $\mathbf{D}_{s}$ is the shear rigidity matrix

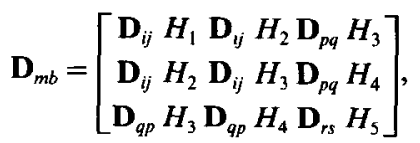

$i=1,2,3,4 ; j=1,2,3,4 ; p=1,2,3,4 ; q=1,2,4 ;$ $r=1,2,4 ; s=1,2,4$;

$$
\begin{gathered}
\left.\mathbf{D}_{s}=\begin{array}{l}
\mathbf{Q}_{m l} H_{1} \mathbf{Q}_{m l} H_{2} \mathbf{Q}_{m l} H_{3} \\
\mathbf{Q}_{m l} H_{2} \mathbf{Q}_{m l} H_{3} \mathbf{Q}_{m l} H_{4} \\
\mathbf{Q}_{m l} H_{3} \mathbf{Q}_{m l} H_{4} \mathbf{Q}_{m l} H_{5}
\end{array}\right], \\
m=6,5 ; l=6,5 .
\end{gathered}
$$

In the above relations, $L$ defines the $L$ th layer, $n$ is the number of layers in a laminate, and

$$
H_{k}=\frac{1}{k}\left(h_{L}^{k}-h_{L+1}^{k}\right), \quad k=1,2,3,4,5 .
$$

By knowing the $\mathbf{B}$ matrix and $\mathbf{D}$ matrix, the element stiffness matrix $\mathbf{K}$ is calculated by using the

$$
\begin{gathered}
{\left[\begin{array}{cccc}
N_{x^{\prime}} & N_{y^{\prime}} & N_{z^{\prime}} & N_{x^{\prime} y^{\prime}} \\
M_{x^{\prime}} & M_{v^{\prime}} & M_{z^{\prime}} & M_{x^{\prime} y^{\prime}} \\
N_{x^{\prime}}^{*} & N_{y^{\prime}}^{*} & N_{x^{\prime} y^{\prime}}^{*}
\end{array}\right]=\sum_{L=1}^{n} \int_{h_{L}+1}^{h_{L}}\left[\begin{array}{cccc}
\sigma_{x^{\prime}} & \sigma_{y} & \sigma_{z^{\prime}} & \tau_{x^{\prime} y^{\prime}} \\
z^{\prime} \sigma_{x^{\prime}} & z^{\prime} \sigma_{y^{\prime}} & z^{\prime} \sigma_{z^{\prime}} & z^{\prime} \tau_{x^{\prime} y^{\prime}} \\
z^{\prime 2} \sigma_{x} & z^{\prime 2} \sigma_{y} & z^{\prime 2} \tau_{x^{\prime} y^{\prime}}
\end{array}\right] \mathrm{d} z} \\
\\
{\left[\begin{array}{lll}
Q_{x^{\prime}} & Q_{y^{\prime}} \\
S_{x^{\prime}} & S_{y^{\prime}} \\
Q_{x^{\prime}}^{*} & Q_{y^{\prime}}^{*}
\end{array}\right]=\sum_{L=1}^{n} \int_{h_{L}, 1}^{h_{L}}\left[\begin{array}{cc}
\tau_{x^{\prime} y^{\prime}} & \tau_{y^{\prime} z^{\prime}} \\
z^{\prime} \tau_{x^{\prime} y^{\prime}} & z^{\prime} \tau_{y^{\prime} z^{\prime}} \\
z^{\prime 2} \tau_{x^{\prime} z^{\prime}} & z^{\prime 2} \tau_{y^{\prime} z^{\prime}}
\end{array}\right] \mathrm{d} z .}
\end{gathered}
$$

After integration, these relations are written in a matrix form which defines the stress-resultant/strain relations of the laminate

$$
\bar{\sigma}^{\prime}=\mathbf{D} \bar{\epsilon}^{\prime}
$$

in which

$$
\begin{aligned}
\overline{\boldsymbol{\sigma}}^{\prime}=\left[N_{x^{\prime}} N_{y^{\prime}} N_{z^{\prime}} N_{x^{\prime} y} M_{y^{\prime}} M_{z^{\prime}} M_{x^{\prime} y^{\prime}} N_{x^{\prime}}^{*}\right. \\
\left.N_{y^{\prime}}^{*} N_{x^{\prime} y^{\prime}}^{*} Q_{x^{\prime}} Q_{y^{\prime}} S_{x^{\prime}} S_{y^{\prime}} Q_{x^{\prime}}^{*} Q_{y^{*}}^{*}\right]^{T}
\end{aligned}
$$

and $\bar{\epsilon}^{\prime}$ is a vector of midsurface strains and is defined in eqn (7b). The rigidity matrix $\mathbf{D}$ can be written as

$$
\mathbf{D}=\left[\begin{array}{cc}
\mathbf{D}_{m b} & \mathbf{0} \\
\mathbf{O} & \mathbf{D}_{s}
\end{array}\right]
$$

where $\mathbf{D}_{m b}$ denotes the membrane, coupling between following

$$
\mathbf{K}=\int_{-1}^{+1} \int_{-1}^{+1} \mathbf{B}^{T} \mathbf{D B}|\mathbf{J}| \mathrm{d} \xi \mathrm{d} \eta .
$$

As the rigidity matrix specifies the properties of the cross-section, the integration for the stiffness matrix is over the midsurface of the shell. The coordinate Jacobian $\mathbf{J}$ is evaluated at the Gauss points lying on the shell midsurface.

\section{NUMERICAL RESULTS AND DISCUSSION}

In order to test the accuracy and the efficiency of the developed algorithm, and to investigate the effects of cross-sectional warping, the following geometric and material properties were used. The values of the principle radii of curvature of the midsurface are denoted by $R_{1}$ and $R_{2}$. 
Data 1. Arc length, $a=b=32.0 \mathrm{~cm}, R_{1}=R_{2}=R=$ varying.

Intensity of UDL, $q=1 \mathrm{~N} / \mathrm{cm}^{2}$.

Face sheets (Graphite/epoxy prepreg system)

$E_{1}=1.308 \times 10^{7} \mathrm{~N} / \mathrm{cm}^{2}, E_{2}=1.06 \times 10^{6} \mathrm{~N} / \mathrm{cm}^{2}$

$G_{12}=G_{13}=6.0 \times 10^{5} \mathrm{~N} / \mathrm{cm}^{2}, G_{23}=3.9 \times 10^{5} \mathrm{~N} / \mathrm{cm}^{2}$,

$v_{12}=v_{13}=0.28, v_{23}=0.34$.

Core (U.S. commercial aluminium honey-comb, $\frac{1}{2}$ in. cell size, 0.003 in. foil).

$G_{23}=1.772 \times 10^{4} \mathrm{~N} / \mathrm{cm}^{2}, G_{13}=5.206 \times 10^{4} \mathrm{~N} / \mathrm{cm}^{2}$.

Data 2. Arc length, $a=b=32.0, q=1.0, R_{1}=R_{2}=R=$ varying.

$E_{1} / E_{2}=E_{1} / E_{3}=25, G_{12}=G_{13}=0.5 E_{2}, G_{23}=0.2 E_{2}$,

$v_{12}=v_{23}=v_{13}=0.25, E_{2}=E_{3}=$ assumed unity.

The load is applied on shell midsurface. It should be noted that unixial and biaxial symmetry exist only in isotropic and cross-ply composite shells, hence quarter of the shell is discretized for cross-ply laminates and full structure is invariably discretized for angle-ply and combination of cross-ply and angle-ply laminated shells. The following boundary conditions were used: simply supported:

$$
\begin{array}{lll}
v_{i}=w_{i}=\beta_{i}=\gamma_{1}=\beta_{i}^{*}=\gamma_{i}^{*}=0 & \text { at } & x=0, a \\
u_{i}=w_{i}=\alpha_{i}=\gamma_{i}=\alpha_{i}^{*}=\gamma_{i}^{*}=0 & \text { at } & y=0, b
\end{array}
$$

clamped supported:

$$
\begin{aligned}
u_{i}=v_{t}=w_{i}=\alpha_{i}=\beta_{i}=\gamma_{i}=\alpha_{i}^{*}=\beta_{i}^{*}=\gamma_{i}^{*}=0 \\
\text { at } x=0, a \text { and } y=0, b
\end{aligned}
$$

symmetric condition:

$$
\begin{array}{lll}
u_{i}=\alpha_{i}=\alpha_{i}^{*}=0 & \text { at } & x=a / 2 \\
v_{i}=\beta_{i}=\beta_{i}^{*}=0 & \text { at } & y=b / 2
\end{array}
$$

In order to investigate the numerical convergence and accuracy of the behaviour of the composite-sandwich shell, a simply supported spherical shell of quarter size is analysed with a uniformly distributed load (UDL) using Data 1. The stacking sequence is $0^{\circ} / 90^{\circ} / 0^{\circ} / 90^{\circ} /$ core $/ 90^{\circ} / 0^{\circ} / 90^{\circ} / 0^{\circ}$, and the thickness of each stiff layer is $0.05 \mathrm{~h}$ and core is $0.6 \mathrm{~h}$. Ratios of radius-to-arc length and are length-to-thickness arc varied. The different Gauss point integration rules are listed in Table 1. Table 2 presents the centre deflection $\left(w \times 10^{-5}\right)$ in centimetres of thick and thin shells with four-, eight- and nine-node quadrilateral shell elements, for different meshes $1 \times 1,2 \times 2$ and $3 \times 3$, using the reduced integration for eight- and ninenode elements and selective integration for four-node elements. Table 3 shows the centre transverse deflection $\left(w \times 10^{-5}\right)$ in centimetres for different Gaussian integration rules, namely reduced, selective and full, with the discretisation of quarter shell by $2 \times 2$ mesh. From these results it follows that the centre deflection varies rapidly, with the ratio $R / a$, for deep shells (i.e. for large ratios of $a / h$ ) than for shallow shells (i.e. for small ratios of $a / h$ ). The behaviour of eight-node serendipity and nine-node Lagrangian elements are almost same. Since the convergence is achieved in $2 \times 2$ mesh only, the nine-node element with reduced integration and $2 \times 2$ mesh are employed in the following examples. It should be noted that, if the radius of the curvature is more, i.e. for $R / a<1.0$, further refinement of mesh size is required. Due to limitation of space those results are not presented.

In order to validate the present theory and element, a problem for which the analytical solution exists is solved. The problem consists of a simply supported composite spherical shell (with Data 2) subjected to a UDL. Table 4 contains the centre transverse deflection $\left(w \times 10^{2}\right)$ for various ratios of radius-to-arc length and for two values of side-to-thickness. A comparison of the present HOST and FOST results with the CFS [7] is made. The results of the present finite element FOST with the CFS are in good agreement with each other but results of the present HOST differ slightly particularly for thick shells. This is due to warping of the cross-section, which is accounted for in HOST.

To show the effectiveness of the present HOST, a clamped composite-sandwich spherical shell with

Table 1. Various Gaussian integration rules employed in evaluation of element properties

\begin{tabular}{lccccc}
\hline $\begin{array}{c}\text { Type of } \\
\text { element }\end{array}$ & Rule & $\begin{array}{c}\text { Membrane terms, and coupling } \\
\text { between inplane and bending terms }\end{array}$ & $\begin{array}{c}\text { Flexure } \\
\text { terms }\end{array}$ & $\begin{array}{c}\text { Shear } \\
\text { terms }\end{array}$ & $\begin{array}{c}\text { Load } \\
\text { vector }\end{array}$ \\
\hline $\begin{array}{l}\text { Eight- and } \\
\text { nine-noded }\end{array}$ & RI & $2 \times 2$ & $2 \times 2$ & $2 \times 2$ & $2 \times 2$ \\
elements & SI & $3 \times 3$ & $3 \times 3$ & $2 \times 2$ & $3 \times 3$ \\
& FI & $3 \times 3$ & $3 \times 3$ & $3 \times 3$ & $3 \times 3$ \\
Four-noded & RI & $1 \times 1$ & $1 \times 1$ & $1 \times 1$ & $1 \times 1$ \\
element & SI & $2 \times 2$ & $2 \times 2$ & $1 \times 1$ & $2 \times 2$ \\
& FI & $2 \times 2$ & $2 \times 2$ & $2 \times 2$ & $2 \times 2$ \\
\hline
\end{tabular}

RI, reduced integration; SI, selective integration, FI, full integration. 
Table 2. Centre deflection (in $\times 10^{-5} \mathrm{~cm}$ ) of a simply supported composite-sandwich spherical shell $\left(0^{\circ} / 90^{\circ} / 0^{\circ} / 90^{\circ} /\right.$ core $\left./ 90^{\circ} / 0^{\circ} / 90^{\circ} / 0^{\circ}\right)$ under a uniformly distributed load and with material Data 1

\begin{tabular}{|c|c|c|c|c|c|c|c|}
\hline \multirow[b]{2}{*}{ Mesh } & \multirow{2}{*}{$R / a^{a / h}$} & \multicolumn{2}{|c|}{ Nine-node $(\mathrm{RI})$} & \multicolumn{2}{|c|}{ Eight-node (RI) } & \multicolumn{2}{|c|}{ Four-node (SI) } \\
\hline & & 10 & 100 & 10 & 100 & 10 & 100 \\
\hline $1 \times 1$ & $\begin{array}{c}1 \\
3 \\
5 \\
10 \\
10^{30}\end{array}$ & $\begin{array}{l}24.43 \\
51.99 \\
56.66 \\
58.88 \\
59.65\end{array}$ & $\begin{array}{l}500.4 \\
04781 \\
11467 \\
26898 \\
48326\end{array}$ & $\begin{array}{l}23.55 \\
48.80 \\
52.98 \\
54.96 \\
55.66\end{array}$ & $\begin{array}{l}477.7 \\
04659 \\
10032 \\
19207 \\
27554\end{array}$ & $\begin{array}{l}17.70 \\
44.34 \\
49.46 \\
51.96 \\
52.84\end{array}$ & $\begin{array}{l}234.3 \\
02255 \\
05649 \\
15145 \\
34234\end{array}$ \\
\hline $2 \times 2$ & $\begin{array}{c}1 \\
3 \\
5 \\
10 \\
10^{30}\end{array}$ & $\begin{array}{l}23.53 \\
50.22 \\
54.66 \\
56.76 \\
57.49\end{array}$ & $\begin{array}{l}459.7 \\
04487 \\
11079 \\
26239 \\
46745\end{array}$ & $\begin{array}{l}24.17 \\
50.53 \\
54.86 \\
56.89 \\
57.60\end{array}$ & $\begin{array}{l}459.4 \\
04478 \\
11049 \\
26135 \\
46491\end{array}$ & $\begin{array}{l}22.16 \\
49.91 \\
54.46 \\
56.60 \\
57.25\end{array}$ & $\begin{array}{l}425.8 \\
04195 \\
10118 \\
24189 \\
44773\end{array}$ \\
\hline $3 \times 3$ & $\begin{array}{c}1 \\
3 \\
5 \\
10 \\
10^{30}\end{array}$ & $\begin{array}{l}23.33 \\
50.84 \\
55.12 \\
57.14 \\
57.84\end{array}$ & $\begin{array}{l}447.8 \\
04494 \\
11079 \\
26217 \\
46662\end{array}$ & $\begin{array}{l}23.69 \\
50.69 \\
55.05 \\
57.09 \\
57.80\end{array}$ & $\begin{array}{l}459.3 \\
04489 \\
11071 \\
26200 \\
46631\end{array}$ & $\begin{array}{l}23.23 \\
50.21 \\
54.68 \\
56.81 \\
57.56\end{array}$ & $\begin{array}{r}396.4 \\
04300 \\
10602 \\
25309 \\
45884\end{array}$ \\
\hline
\end{tabular}

RI, reduced integration; SI, selective integration.

Table 3. Centre deflection (in $\times 10^{-5} \mathrm{~cm}$ ) of a simply supported nine-layered symmetric compositesandwich spherical shell under uniformly distributed load, material Data 1 and $2 \times 2$ mesh in a quarter shell

\begin{tabular}{|c|c|c|c|c|c|c|c|c|c|c|}
\hline \multirow[b]{2}{*}{$a / h$} & \multirow[b]{2}{*}{$R / a$} & \multicolumn{3}{|c|}{ Nine-node } & \multicolumn{3}{|c|}{ Eight-node } & \multicolumn{3}{|c|}{ Four-node } \\
\hline & & RI & SI & FI & RI & SI & FI & RI & SI & FI \\
\hline \multirow{5}{*}{10} & 1 & 23.53 & 23.81 & 23.73 & 24.17 & 23.75 & 23.68 & 23.82 & 22.16 & 21.28 \\
\hline & 3 & 50.22 & 50.69 & 50.61 & 50.53 & 50.78 & 50.48 & 51.94 & 49.91 & 43.18 \\
\hline & 5 & 54.66 & 55.07 & 54.98 & 54.86 & 54.97 & 54.84 & 56.69 & 54.46 & 46.65 \\
\hline & 10 & 56.76 & 57.14 & 57.03 & 56.89 & 57.00 & 56.89 & 58.94 & 56.60 & 48.27 \\
\hline & $10^{30}$ & 57.49 & 57.86 & 57.75 & 57.60 & 57.71 & 57.61 & 59.64 & 57.25 & 48.83 \\
\hline \multirow{5}{*}{100} & 1 & 459.7 & 452.2 & 460.5 & 459.4 & 453.0 & 460.0 & 413.1 & 425.8 & 351.72 \\
\hline & 3 & 04487 & 04463 & 04548 & 04478 & 04453 & 04534 & 04390 & 04195 & 01478 \\
\hline & 5 & 11079 & 10993 & 11019 & 11049 & 10962 & 10956 & 10659 & 10118 & 01876 \\
\hline & 10 & 26239 & 26101 & 25676 & 26135 & 25995 & 25395 & 25523 & 24189 & 02112 \\
\hline & $10^{30}$ & 46745 & 46723 & 45156 & 46491 & 46470 & 44375 & 47129 & 44773 & 02204 \\
\hline
\end{tabular}

RI, reduced integration; SI, selective integration; FI, full integration.

Data 1 and stacking sequence as $0^{\circ} / 45^{\circ} /$ core $/ 60^{\circ} / 90^{\circ}$ and subjected to a UDL is analysed. A whole shell is discretized with $4 \times 4$ mesh. Thickness of each stiff layer is $0.05 h$ and core material is $0.8 h$. Table 5 shows the centre transverse deflection $\left(w \times 10^{-4}\right)$ in centimetres for different ratios of $R / a$ and $a / h$. From this table, it is seen that, maximum difference between FOST and HOST is about $18 \%$ in the case of moderately thick shell $(a / h=10)$ and about $1 \%$ in thin shells $(a / h=100)$. This is due to a realistic representation of the cross-sectional deformation and consideration of the complete stress-strain law in the present HOST.

\section{CONCLUSIONS}

The evaluations using the present higher-order shear deformable shell theory (HOST) show considerable warping of the transverse cross-section for composite-sandwich shells. This true behaviour is not possible to model with a first-order shear deformation theory (FOST). The refined theory with a $C^{0}$ continuous finite element model presented here is essential for reliable analyses of fibre-reinforced orthotropic and anisotropic composite and sandwich shell structures. In contrast to the classical shear deformation theories, the present HOST does not require a shear correction coefficient, owing to more realistic representation of the cross-sectional deformation and consideration of the complete threedimensional Hooke's law. Further, the finite element formulation presented here has enhanced the practical applicability of such a theoretical development.

Acknowledgement-Partial support of this research by the Aeronautics Research and Development Board, Ministry of Defence, Government of India, through its Grant Nos Aero/RD-134/100/88-89/518 and Aero/RD-134/100/77$89 / 534$ is gratefully acknowledged. 
Table 4. Centre deflection of a simply supported composite spherical shell with Data 2

\begin{tabular}{|c|c|c|c|c|c|}
\hline \multirow[b]{2}{*}{$R / a$} & \multirow[b]{2}{*}{$a / h$} & \multicolumn{2}{|c|}{$0^{\circ} / 90^{\circ}$} & \multicolumn{2}{|c|}{$0^{\circ} / 90^{\circ} / 90^{\circ} / 0^{\circ}$} \\
\hline & & 10 & 100 & 10 & 100 \\
\hline \multirow{3}{*}{$10^{30}$} & HOST & 8.1297 & 5452.3 & 3.1215 & 2189.2 \\
\hline & FOST & 6.2426 & 5445.5 & 3.2864 & 2191.0 \\
\hline & CFS & 6.2300 & 5433.6 & 3.2803 & 2186.59 \\
\hline \multirow{3}{*}{10} & HOST & 7.8232 & 1767.4 & 3.0749 & 1188.0 \\
\hline & FOST & 6.0817 & 1770.9 & 3.2375 & 1188.4 \\
\hline & CFS & 6.1008 & 1773.6 & 3.2451 & 1190.6 \\
\hline \multirow{3}{*}{5} & HOST & 7.1138 & 555.46 & 2.9356 & 488.58 \\
\hline & FOST & 5.6439 & 558.59 & 3.0986 & 488.60 \\
\hline & CFS & 6.3820 & 561.12 & 3.1439 & 491.45 \\
\hline \multirow{3}{*}{4} & HOST & 6.6687 & 360.11 & 2.8189 & 335.35 \\
\hline & FOST & 5.3536 & 362.84 & 3.0015 & 335.34 \\
\hline & CFS & 5.4988 & 365.18 & 3.0719 & 337.88 \\
\hline \multirow{3}{*}{3} & HOST & 5.8782 & 201.74 & 2.8298 & 197.82 \\
\hline & FOST & 4.8147 & 204.02 & 2.8098 & 197.80 \\
\hline & CFS & 5.0364 & 206.11 & 2.9268 & 199.87 \\
\hline \multirow{3}{*}{2} & HOST & 4.3869 & 87.806 & 2.3181 & 89.462 \\
\hline & FOST & 3.7211 & 89.784 & 2.3678 & 89.445 \\
\hline & CFS & 4.0537 & 91.360 & 2.5765 & 91.008 \\
\hline
\end{tabular}

\section{REFERENCES}

1. V. Z. Vlasov, Basic differential equations in general theory of elastic shells. NASA TM-1241 (1951).

2. S. Ambartsumyan, Theory of anisotropic shell. NASA TT F-118 (1964).

3. P. M. Naghdi, On a variational theorem in elasticity and its application to shell theory. Trans ASME, J. Appl. Mech. 31, 647-653 (1964).

4. T. M. Hsu and J. T. S. Wang, A theory of laminated cylindrical shells consisting of layers of orthotropic laminate. AlAA J. 8, 2141-2146 (1970).

5. J. A. Zukas and J. R. Vinson, Laminated transversely isotropic cylindrical shells. Trans ASME, J. Appl. Mech. 38, 400-407 (1971).

6. S. B. Dong and F. K. W. Tso, On a laminated orthotropic shell including shear deformation. Trans ASME, J. Appl. Mech. 39, 1091-1097 (1972).

7. J. N. Reddy, Exact solutions of moderately thick laminated shells. ASCE, J. Engng Mech. 110, 794-809 (1984).

8. C. W. Bert, Analysis of shells. In Structural Design and Analysis, pp. 207-258. Part I, (Edited by C. C. Chamis), Vol. 7 of Composite Materials. John Wiley, New York (1974).

9. J. M. Whitney and C. T. Sun, A refined theory for laminated anisotropic cylindrical shells. Trans $A S M E$, J. Appl. Mech. 41, 471-476 (1974).

10. G. E. O. Widera and D. L. Logan, Refined theories for non-homogeneous anisotropic cylindrical shells, Part I: derivation, Part II: applications. ASCE, J. Engng Mech. 106, 1053-1090 (1980).

11. T. Kant, On finite element discretization of a higherorder shell theory. In The Mathematics of Finite Elements and Applications IV (MAFELAP 1981), pp. 209-217 (Edited by J. R. Whiteman). Academic Press, London (1982).

12. A. V. Krishna Murthy and T. S. R. Reddy, A higher order theory of laminated composite cylindrical shells. J. Aero. Soc. India 161-171 (1986).
Table 5. Centre deflection of a clamped unsymmetric $\left(0^{\circ} / 45^{\circ} /\right.$ core $\left./ 60^{\circ} / 90^{\circ}\right)$ composite-sandwich spherical shell

\begin{tabular}{|c|c|c|c|}
\hline \multirow[b]{2}{*}{$R / a$} & \multirow[b]{2}{*}{$a / h$} & \multicolumn{2}{|c|}{$0^{\circ} / 45^{\circ} /$ core $/ 60^{\circ} / 90^{\circ}$} \\
\hline & & 10 & 100 \\
\hline $10^{30}$ & $\begin{array}{l}\text { HOST } \\
\text { FOST }\end{array}$ & $\begin{array}{c}7.0894 \\
5.7593 \\
(-18.7)\end{array}$ & $\begin{array}{l}3218.3 \\
3212.4 \\
(0.18)\end{array}$ \\
\hline 10 & $\begin{array}{l}\text { HOST } \\
\text { FOST }\end{array}$ & $\begin{array}{c}6.9199 \\
5.6097 \\
(-18.9)\end{array}$ & $\begin{array}{l}1355.2 \\
1345.5 \\
(0.05)\end{array}$ \\
\hline 5 & $\begin{array}{l}\text { HOST } \\
\text { FOST }\end{array}$ & $\begin{array}{c}6.4214 \\
5.2086 \\
(-18.9)\end{array}$ & $\begin{array}{c}459.99 \\
460.06 \\
(-0.01)\end{array}$ \\
\hline 2 & $\begin{array}{l}\text { HOST } \\
\text { FOST }\end{array}$ & $\begin{array}{c}4.2027 \\
3.4677 \\
(-17.5)\end{array}$ & $\begin{array}{l}63.568 \\
63.390 \\
(0.28)\end{array}$ \\
\hline 1 & $\begin{array}{l}\text { HOST } \\
\text { FOST }\end{array}$ & $\begin{array}{c}1.8481 \\
1.5809 \\
(-14.4)\end{array}$ & $\begin{array}{l}15.751 \\
15.561 \\
(1.22)\end{array}$ \\
\hline 0.7 & $\begin{array}{l}\text { HOST } \\
\text { FOST }\end{array}$ & $\begin{array}{c}1.0236 \\
0.9325 \\
(-8.89)\end{array}$ & $\begin{array}{l}9.2800 \\
9.1635 \\
(1.27)\end{array}$ \\
\hline
\end{tabular}

Values in parentheses indicate the percentage difference with respect to HOST.

13. T. Kant, A consistent higher-order theory for laminated composite shells. In Advances in Aerospace Structures and Allied Fields, pp. 61-69 (Edited by T. K. Varadan). Mass Prints, Madras (1988).

14. A. K. Noor and P. L. Rarig Three-dimensional solutions of laminated cylinders. Comp. Meth. Appl. Mech. Engng 3, 319-334 (1974).

15. K. Chandrashekhara and A. Bhimaraddi, Shell core method for the analysis of a long circular cylindrical sandwich shell subjected to axisymmetric loading. Struct. Mech. 10, 329-351 (1982-1983).

16. Mallikarjuna, Refined theories with $C^{0}$ finite elements for free vibration and transient dynamics of anisotropic composite-sandwich plates. Ph.D. thesis, Indian Institute of Technology, Bombay (1988).

17. Mallikarjuna and T. Kant, Finite element transient response of composite and sandwich plates with a refined higher-order theory. ASME, J. Appl. Mech. 57, 1084-1086 (1990).

18. Mallikarjuna and T. Kant, Free vibration of symmetrically laminated plates using a higher-order theory with finite element technique. Int. J. Numer. Meth. Engng 28, 1875-1889 (1989).

19. T. Kant and Mallikarjuna, A higher-order theory for free vibration of unsymmetrically laminated composite and sandwich plates-finite element evaluations. Comput. Struct. 32, 1125-1132 (1989).

20. T. Kant and Mallikarjuna, Vibrations of unsymmetrically laminated plates analysed by using a higher-order theory with $C^{0}$ finite element formulation. $J$. Sound Vibr. 134, 1-16 (1989).

21. S. Ahmad, B. M. Irons and O. C. Zienkiewicz, Analysis of thick and thin shell structures by curved elements. Int. J. Numer. Meth. Engng 2, 419-451 (1970),

22. T. Belytschko, H. Stolarski, W. K. Liu, N. Carpenter and J. S. J. Ong, Stress projection for membrane and shear locking in shell finite elements. Comput. Meth. Appl. Mech. Engng 51, 221-258 (1985).

23. R. V. Milford and W. C. Schnobrich, Degenerated isoparametric finite elements using explicit integration. Int. J. Numer. Meth. Engng 23, 133-154 (1986). 
24. T. Kant and D. Datye, Finite elements available for the analysis of curved thin walled structures. In Finite Element Applications to Thin-walled Structures, Chap. 1, pp. 1-40 (Edited by J. W. Bull). Elsevier (1990).
25. Mallikarjuna and T. Kant, Transient response of isotropic, orthotropic and anisotropic fibre reinforced composite shells (to be published). 